PROCEEDINGS OF THE

AMERICAN MATHEMATICAL SOCIETY

Volume 125, Number 9, September 1997, Pages 2649-2650

S $0002-9939(97) 03834-3$

\title{
CONSTRUCTION OF INVARIANT CURVES FOR SINGULAR HOLOMORPHIC VECTOR FIELDS
}

\author{
J. CANO
}

(Communicated by Mary Rees)

\begin{abstract}
Camacho and Sad proved the existence of invariant analytic curves for germs of singular holomorphic foliations $\mathcal{F}$ over a two dimensional complex analytic variety $M$. Their proof is only of existential nature. Here we provide a simple constructive proof by giving criteria to choose a singular point at each blowing-up that follows an analytic invariant curve.
\end{abstract}

Our algorithm is founded on the stability by blowing-up of the property $(\star)$ introduced in the following definition.

Definition. Consider a singular holomorphic foliation $\mathcal{F}$ over a two dimensional complex analytic variety $M$, a normal crossings divisor $E$ over $M$ and a point $q \in E$. We say that the triple $(\mathcal{F}, E, q)$ has the property $(\star)$ if and only if one of the following properties holds:

$(\star)$-1: The point $q$ lies exactly in one irreducible component $S$ of $E$, which is invariant for $\mathcal{F}$ and the index $i_{q}(\mathcal{F}, S) \notin \mathbb{Q}(\geq 0)=\{r \in \mathbb{Q} ; r \geq 0\}$.

( $\star$ )-2: The point $q$ lies in two irreducible components $S_{+}$and $S_{-}$of $E$ (call this point a "corner"), both are invariant curves and there is a real number $a>0$ such that:

$$
\begin{aligned}
& i_{q}\left(\mathcal{F}, S_{+}\right) \quad \in \mathbb{Q}_{(\leq-a)}=\{r \in \mathbb{Q} ; r \leq-a\}, \\
& i_{q}\left(\mathcal{F}, S_{-}\right) \quad \notin \mathbb{Q}_{(\geq-1 / a)}=\{r \in \mathbb{Q} ; r \geq-1 / a\} .
\end{aligned}
$$

( $\star$-3: The point $q$ lies exactly in one irreducible component $S$ of $E$, it is a nonsingular point of $\mathcal{F}$ and $S$ is transversal to $\mathcal{F}$ at $q$.

(The definition and basic properties of the index can be found in [1]).

Remark. If we have the property $(\star)-2$, then $q$ is not a simple (irreducible) singularity. If we have either $(\star)-1$ and $q$ is a simple singularity or $(\star)-3$, then there is a nonsingular analytic invariant curve $\Gamma$ through $q$ transversal to $E$.

Theorem. Assume that $(\mathcal{F}, E, q)$ satisfies either $(\star)-1$ or $(\star)-2$. Consider the blowing-up $\pi: M^{\prime} \rightarrow M$ at the point $q$. Let $\mathcal{F}^{\prime}$ be the strict transform of $\mathcal{F}$ by $\pi$. Put $D=\pi^{-1}(q)$ and $E^{\prime}=\pi^{-1}(E)$. Then there is a point $q^{\prime} \in D$ such that $\left(\mathcal{F}^{\prime}, E^{\prime}, q^{\prime}\right)$ satisfies the property $(\star)$.

Received by the editors October 24, 1995 and, in revised form, March 19, 1996.

1991 Mathematics Subject Classification. Primary 34A05; Secondary 32S65.

This work was supported by the I.A.S. under NSF grant \# DMS-9304580.

(C)1997 American Mathematical Society 
Proof. If $\pi$ is a dicritical blowing-up we immediately get a point $q^{\prime} \in D$ such that $\left(\mathcal{F}^{\prime}, E^{\prime}, q^{\prime}\right)$ satisfies the property $(\star)-3$.

Assume that $\pi$ is non dicritical and hence $D$ is an invariant curve for $\mathcal{F}^{\prime}$.

Consider first the case that $(\mathcal{F}, E, q)$ satisfies $(\star)-1$. Let $S^{\prime}$ be the strict transform of $S$ by $\pi$ and put $\left\{q^{\prime}\right\}=D \cap S^{\prime}$. Let $p_{1}, \ldots, p_{s}$ be the singularities of $\mathcal{F}^{\prime}$ on $D \backslash\left\{q^{\prime}\right\}$. Suppose that $\left(\mathcal{F}^{\prime}, E^{\prime}, p_{i}\right)$ does not satisfy $(\star)-1$ for any $i=1, \ldots, s$. Then we have that

$$
i_{q^{\prime}}\left(\mathcal{F}^{\prime}, D\right)=-1-\sum_{i=1}^{s} i_{p_{i}}\left(\mathcal{F}^{\prime}, D\right) \in \mathbb{Q}_{(\leq-1)} .
$$

Since $i_{q^{\prime}}\left(\mathcal{F}^{\prime}, S^{\prime}\right)=i_{q}(\mathcal{F}, S)-1 \notin \mathbb{Q}_{(\geq-1)}$, then $\left(\mathcal{F}^{\prime}, E^{\prime}, q^{\prime}\right)$ satisfies $(\star)-2$.

Consider now the case that $(\mathcal{F}, E, q)$ satisfies $(\star)-2$. Let $S_{+}^{\prime}$ and $S_{-}^{\prime}$ be the strict transforms by $\pi$ of $S_{+}$and $S_{-}$respectively. Let $p_{1}, \ldots, p_{s}$ be the singularities of $\mathcal{F}^{\prime}$ on $D \backslash\left\{q_{+}, q_{-}\right\}$, where $q_{+}=D \cap S_{+}^{\prime}$ and $q_{-}=D \cap S_{-}^{\prime}$. If $\left(\mathcal{F}^{\prime}, E^{\prime}, p_{i}\right)$ does not satisfy $(\star)-1$ for $i=1, \ldots, s$, then

$$
i_{q_{+}}\left(\mathcal{F}^{\prime}, D\right)+i_{q_{-}}\left(\mathcal{F}^{\prime}, D\right)=-1-\sum_{i=1}^{s} i_{p_{i}}\left(\mathcal{F}^{\prime}, D\right) \in \mathbb{Q}_{(\leq-1)}
$$

Since $(\mathcal{F}, E, q)$ satisfies the property $(\star)-2$ we have that

$$
\begin{aligned}
& i_{q_{+}}\left(\mathcal{F}^{\prime}, S_{+}^{\prime}\right)=i_{q}\left(\mathcal{F}, S_{+}\right)-1 \in \mathbb{Q}_{(\leq-(a+1))}, \\
& i_{q_{-}}\left(\mathcal{F}^{\prime}, S_{-}^{\prime}\right)=i_{q}\left(\mathcal{F}, S_{-}\right)-1 \notin \mathbb{Q}_{\left(\geq-\frac{a+1}{a}\right)} .
\end{aligned}
$$

If $\left(\mathcal{F}^{\prime}, E^{\prime}, q_{+}\right)$does not satisfy $(\star)-2$ then $i_{q_{+}}\left(\mathcal{F}^{\prime}, D\right) \in \mathbb{Q}_{\left(\geq-\frac{1}{a+1}\right)}$. By $(1) i_{q_{-}}\left(\mathcal{F}^{\prime}, D\right)$ $\in \mathbb{Q}_{\left(\leq-\frac{a}{a+1}\right)}$ and thus $\left(\mathcal{F}^{\prime}, E^{\prime}, q_{-}\right)$satisfies $(\star)-2$.

To get an analytic invariant curve $\Gamma$ for $\mathcal{F}$ at $q$ we proceed as follows. After the blowing-up with center $q$ we take a point $p_{1}$ in the exceptional divisor $E_{1}$ with the property $(\star)$ : if the blowing-up is dicritical we get $(\star)-3$ and the algorithm stops, otherwise we get $(\star)-1$ since the sum of the indices is -1 . Repeat. By reduction of singularities, in a finite number of steps we get either $(\star)-3$ or an irreducible singularity satisfying $(\star)$. This gives an analytic invariant curve $\Gamma^{\prime}$ transversal to the divisor that projects over $\Gamma$.

\section{REFERENCES}

[1] C. Camacho and P. Sad. Invariant varieties through singularities of holomorphic vector fields, Ann. of Math., 115, (1982) 579-595. MR 83m:58062

Facultad de Ciencias, Universidad de Valladolid, 47005-Valladolid, Spain

E-mail address: jcano@cpd.uva.es 